\title{
Determination of Se(IV) and Se(VI) in Environmental Water Samples Using Solidified Floating Organic Drop Microextraction Coupled With Electrothermal Vaporization ICP-MS
}

\author{
Shizhong Chen*, Shengping Zhu, and Dengbo Lu \\ College of Chemical and Environmental Engineering, Wuhan Polytechnic University, \\ 68 Xuefu South Road Changqing Garden, Wuhan 430023, P.R. China
}

\section{INTRODUCTION}

In recent years, there has been a growing interest in the determination of chemical forms of selenium (Se) in environmental and biological samples due to their different beneficial or toxic effects. The investigation showed that Se has a very narrow concentration range from sufficiency to deficiency and toxicity (1). Se deficiency can lead to heart disease, hypothyroidism, and a weakened immune system, but excess exposure to Se often results in gastrointestinal disturbances, hair and nail changes, and neurologic manifestations $(2,3)$. It is worth noting that the nutritional and toxicological impact of Se depends on its chemical forms (4). In general, inorganic species of Se are more toxic than its organic forms, and the toxicity of $\mathrm{Se}(\mathrm{VI})$ is more severe than Se(IV) $(5,6)$. Therefore, it is necessary not only to determine the total Se in different environmental samples but also to determine the speciation of Se, especially its inorganic forms with higher toxicity.

The speciation of elements has been generally achieved by combining a very effective separation method with a sensitive detection technique. Many separation methods such as coprecipitation, solvent extraction, cloud point extraction, solid phase extraction, hydride generation, capillary electrophoresis, and chromatography have been used for elemental speciation (7-13).

*Corresponding author.

E-mail: chenshizhong62@163.com

\begin{abstract}
A new method was developed for the determination of Se(IV) and $\mathrm{Se}(\mathrm{VI})$ in environmental water samples by inductively coupled plasma mass spectrometry (ICP-MS), using solidified floating organic drop microextraction (SFODME) coupled with electrothermal vaporization (ETV) as a sample introduction procedure. Ammonium pyrroinedithiocarbamate (APDC) was used as both the chelating reagent in SFODME and the chemical modifier in ETV. Se(IV) and $\mathrm{Se}(\mathrm{VI})$ were separated at the $\mathrm{pH}$ range of 2.0-5.0. Total Se was determined after reducing $\mathrm{Se}(\mathrm{VI})$ to Se(IV). The concentration of Se(VI) was based on subtracting Se(IV) from total Se. The main factors affecting the formation and vaporization of the $\mathrm{Se}(\mathrm{IV}) / \mathrm{Se}$ (VI)-APDC complex were investigated in detail. Under the optimum conditions, the detection limits for Se(IV) and $\mathrm{Se}(\mathrm{VI})$ were 0.26 and $0.34 \mathrm{pg}$ $\mathrm{mL}^{-1}$, respectively. The relative standard deviations were less

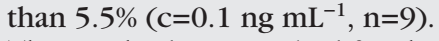
This method was applied for the determination of Se(IV) and $\mathrm{Se}(\mathrm{VI})$ in natural water samples with the recovery for the spiked samples in the range of 93.2-106\%.
\end{abstract}

However, these techniques still have some disadvantages, including large consumption of reagent, multistage operation, unsatisfactory enrichment factor, and lengthy separation. To overcome these problems, a new separation technique, namely solidified floating organic drop microextraction (SFODME), was developed as a powerful sample pretreatment tool prior to detection (14). Owing to its unique advantages of simplicity, speed, low cost, consumption of small organic solvent, and high enrichment factor, SFODME has been successfully employed for the determination of trace elements (15-19). However, to the best of our knowledge, the use of SFODME for the separation and preconcentration of $\mathrm{Se}(\mathrm{VI})$ and Se(IV) has not been reported in the literature.

It is well known that inductively coupled plasma mass spectrometry (ICP-MS) has been widely used for the determination of trace elements owing to its high sensitivity, wide linear dynamic range, rapid multielement detection capability, and absence of line-rich spectral interferences encountered in inductively coupled plasma atomic emission spectrometry (ICP-OES). It is predicted that SFODME coupled with ICP-MS may be a powerful detection tool for elements. Unfortunately, the liquid nebulization sample introduction procedure for conventional inductively coupled plasma mass spectrometry (ICP-MS) is not compatible with SFODME because of its large sample consumption. Therefore, much work has been done on SFODME for the separation and preconcentration of trace elements by atomic absorption spectrometry, but only few researchers reported using ICP-MS $(20,21)$.

Electrothermal vaporization (ETV), as a sample introduction device for ICP-MS, has the merits of 
high introduction efficiency, small sample requirement, and low absolute detection limit. Moreover, use of a chemical modifier in ETV could greatly improve the analytical performance of the method (2225). As described previously, SFODME is a miniaturized sample pretreatment technique, and ETV is a micro-amount sample introduction device. Therefore, SFODME coupled with ETV for ICP-MS may be a perfect combination. However, studies on SFODME-ETV-ICP-MS for elemental speciation have received little attention so far.

The aim of this work is to use ammonium pyrroinedithiocarbamate (APDC) as both a chelating reagent in SFODME and a chemical modifier in ETV for the separation, preconcentration, and volatilization of Se(IV) and Se(VI) prior to their determination by ICP-MS. The main factors affecting separation, preconcentration, and determination of the analytes were investigated in detail. The developed method was applied for the determination of $\mathrm{Se}(\mathrm{IV})$ and Se(VI) in natural water samples with satisfactory results.

\section{EXPERIMENTAL}

\section{Instrumentation}

An X-7 ICP-MS system (Thermo Elemental Corporation, USA), equipped with a modified commercially available WF-4C graphite furnace (Beijing Second Optics, P.R. China) as an electrothermal vaporizer, was used for determination of the analytes. The operating parameters of ICP-MS were optimized with a concentric glass nebulizer prior to connection with the ETV device. A pyrolytic graphite tube was used throughout this work. The working conditions of ETV-ICP-MS are summarized in Table $\mathrm{I}$. The $\mathrm{pH}$ value of the solution was controlled with a pH meter (Thermo Orion Corporation, USA) supplied with a combined electrode.

\section{Standard Solutions and Reagents}

All reagents used in this study were of the highest purity available or at least of analytical grade. High purity deionized water, obtained from a Milli-Q ${ }^{\circledR}$ A10 system (Millipore Corporation, USA), was used throughout this work. The stock standard solutions of Se(IV) and Se(VI) $\left(1.0 \mathrm{mg} \mathrm{mL}^{-1}\right)$ were prepared by dissolving the appropriate amounts of sodium selenite and sodium selenate (Shanghai Reagent Factory, Shanghai, P.R. China) in high purity deionized water. Working solutions were prepared daily by appropriate dilution of the stock solutions. An APDC solution was prepared by dissolving the proper amount of APDC (Shanghai Reagent Factory, P.R. China) in 1-undecanol (Shanghai Reagent Factory, P.R. China). All glass and polypropylene wares were kept in $2.0 \mathrm{~mol} \mathrm{~L}^{-1}$ $\mathrm{HNO}_{3}$ for at least one night, then rinsed with $0.1 \mathrm{~mol} \mathrm{~L}^{-1} \mathrm{HNO}_{3}$ and

\section{Atomic Apectroscopy 1 Vol. 36(3), May/June 2015}

subsequently with doubly distilled water before use.

\section{Sample Preparation}

Natural water samples (collected from Wuhan, P.R. China) were filtered through a $0.45 \mu \mathrm{m}$ membrane filter immediately and then kept at $4{ }^{\circ} \mathrm{C}$ before use. The filtrate mentioned above was directly used for the determination of Se(IV) without digestion. To obtain the concentration of $\mathrm{Se}(\mathrm{VI})$, the filtrate was heated in a gentle boiling water bath for 20 minutes after the addition of $5.0 \mathrm{~mol} \mathrm{~L}^{-1} \mathrm{HCl}$ to reduce $\mathrm{Se}(\mathrm{VI})$ into Se(IV) according to the literature (26). The obtained solution was used for the determination of the sum of Se(VI) and Se(IV). The content of Se(VI) was calculated by subtracting Se(IV) from the sum. The blanks were prepared in exactly the same way.

TABLE I

Operating Parameters for ETV-ICP-MS

\begin{tabular}{ll}
\hline ICP-MS Plasma & \\
\hline Plasma Power & $1300 \mathrm{~W}$ \\
Plasma Argon Flow Rate & $14.3 \mathrm{~L} \mathrm{~min}^{-1}$ \\
Auxiliary Argon Flow Rate & $0.75 \mathrm{~L} \mathrm{~min}^{-1}$ \\
Nebulizer Argon Flow Rate & $0.95 \mathrm{~L} \mathrm{~min}^{-1}$ \\
Sampler Orifice (nickel) & $1.1 \mathrm{~mm}$ \\
Skimmer Orifice (nickel) & $0.7 \mathrm{~mm}$ \\
Acquisition Mode & Peak jumping \\
Number of Sweeps & 100 \\
Dwell Time & $10 \mathrm{~ms}$ \\
Acquisition Time & $40 \mathrm{~s}$ \\
Number of Measurements per Peak & 3 \\
Isotope & $82 \mathrm{Se}$ \\
\hline ETV & \\
\hline Sample Volume & $10 \mu \mathrm{L}$ \\
Carrier Gas Flow Rate & $0.43 \mathrm{~L} \mathrm{~min}{ }^{-1}$ \\
Drying Step & $200{ }^{\circ} \mathrm{C}$, ramp $10 \mathrm{~s}$, hold $30 \mathrm{~s}$ \\
Vaporization Step & $1300{ }^{\circ} \mathrm{C}$, hold $4 \mathrm{~s}$ \\
Clear-out Temperature & $2500^{\circ} \mathrm{C}$ \\
\hline
\end{tabular}




\section{SFODME Extraction}

The SFODME device used in this work for extraction of the analytes was similar to that described in the literature (27). The sample solution containing analytes was adjusted to the desired $\mathrm{pH}$ values using diluted $\mathrm{HNO}_{3}$ solution. Then $10 \mathrm{~mL}$ of the resulting solution was transferred into an $11-\mathrm{mL}$ vial, and a stir bar and $20 \mu \mathrm{L}$ of APDC in 1-dodecanol were added. The magnetic stirrer was turned on, and the solution was stirred for a fixed time. In this step, analytes react with APDC to form hydrophobic complexes which are extracted into 1-undecanol. After the extraction process, the sample vial was transferred into an ice bath until the organic solvent was solidified. The solidified solvent was then transferred into a conical vial where it melted immediately at room temperature. The extract was diluted to $100 \mu \mathrm{L}$ with tetrahydrofuran (THF). Finally, $10 \mu \mathrm{L}$ of the extract was injected into the graphite tube for ETVICP-MS determination.

\section{ETV-ICP-MS Determination}

After the ETV unit had been connected to the ICP-MS, $10 \mu \mathrm{L}$ of the analytes were injected into the graphite furnace. During the drying and charring process, the dosing hole of the graphite furnace was kept open to remove water and organic vapor. It was then sealed with a graphite probe for 5-10 seconds prior to the vaporization step. The vaporized analytes were swept into the plasma excitation source by a carrier gas, and the peak-hop transient mode for data acquisition was used for determination of the analytes.

\section{RESULTS AND DISCUSSION}

\section{Signal Profiles of Se(IV) and Se(VI) With and Without APDC}

To separate Se(IV) from Se(VI) in the ETV, the vaporization characteristics of Se(IV) and Se(VI) were investigated with and without APDC as the chemical modifier. Figure 1 shows the typical signal profiles of $\mathrm{Se}(\mathrm{IV})$ and $\mathrm{Se}(\mathrm{VI})$. It can be seen from this figure that there is an intensive and sharp signal profile for $\mathrm{Se}(\mathrm{IV})$ in the presence of APDC (c). On the contrary, no signal was recorded for Se(IV) without APDC (b). It is worth noting that the signal of Se(VI) was not detected with APDC (d). In addi- tion, the blank signal of the corresponding solution was not obtained in this experiment (a). The facts mentioned above show that Se(IV) and APDC form a complex that can be extracted into the organic phase of 1-dodecanol, whereas Se(VI) does not react with APDC to form the complex at the same conditions. Thus, in this work, APDC and 1-dodecanol were used as both a chelating reagent and an extraction solvent for the separation of Se(IV) from Se(VI).

\section{Effect of pH on Formation of Se(IV)/Se(VI)-APDC Complex}

For SFODME, the $\mathrm{pH}$ value plays an essential role in the Se-APDC complex formation and subsequent extraction with 1-dodecanol since it directly affects the existing form of the analytes and the chelating reagent. Thus, the effects of sample $\mathrm{pH}$ on the signal intensity of Se(VI) and Se(IV) were investigated in the $\mathrm{pH}$ range of 1.0-7.0. Figure 2 shows that maximal signal intensity of Se(IV) was obtained in the $\mathrm{pH}$ range of 2.0-5.0, whereas no signal of Se(VI) was detected in the same $\mathrm{pH}$ range. Therefore, $\mathrm{pH} 4.0$ was selected for subsequent experiments and real sample analysis.

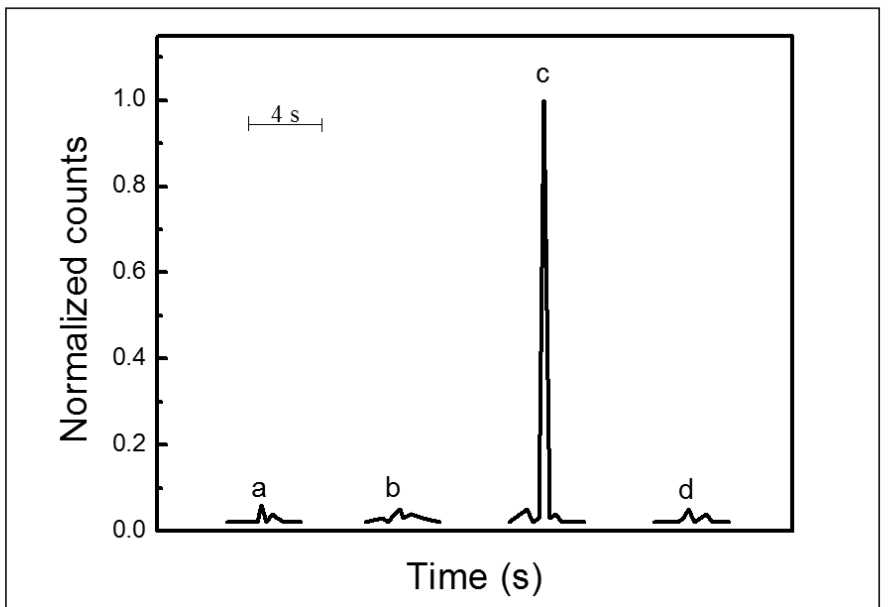

Fig. 1. Signal profiles for Se(IV) and Se(VI) with and without APDC. a: APDC in 1-undecanol and THF; b: Se(IV) in 1-undecanol and THF; : Se(IV) with APDC in 1-undecanol and THF; d: Se(VI) with APDC in 1-undecanol and THF. Se(IV)

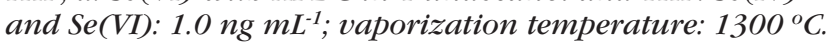

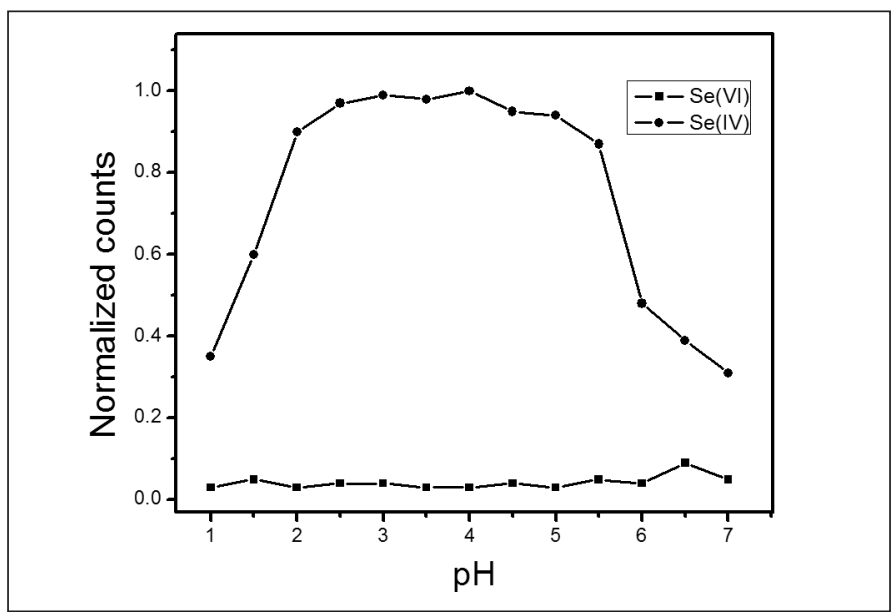

Fig. 2. Effect of $p H$ on the formation of $\mathrm{Se}(\mathrm{IV}) / \mathrm{Se}(\mathrm{VI})-\mathrm{APDC}$ complex. Se(IV) and Se(VI): $1.0 \mathrm{ng} \mathrm{mL}^{-1}$. 


\section{Atomic Apectroscopy \\ 1 Vol. 36(3), May/June 2015}

\section{Effect of APDC Concentration}

The influence of APDC concentration on the formation of the $\mathrm{Se}(\mathrm{IV}) / \mathrm{Se}$ (VI)-APDC complex was studied in the APDC concentration range of $0.1 \times 10^{-2}-3.0 \times 10^{-2} \mathrm{~mol} \mathrm{~L}^{-1}$. The results in Figure 3 show that the signal intensity of the analyte increased with an increase in APDC concentration from $0.1 \times 10^{-2}$ to $1.5 \times 10^{-2} \mathrm{~mol} \mathrm{~L}^{-1}$, and then remained nearly constant when the amount of APDC exceeded $1.5 \times 10^{-2} \mathrm{~mol} \mathrm{~L}^{-1}$. Taking into account that APDC could be consumed by interfering ions, the concentration of $2.0 \times 10^{-2} \mathrm{~mol} \mathrm{~L}^{-1}$ APDC was used in subsequent experiments.

\section{Extraction Time and Temperature}

Extraction time is an important factor influencing the extraction efficiency and analysis speed. The effect of extraction time on signal intensity of Se(IV) was investigated in the time range of 10-50 minutes. The results showed that the signal intensity of Se(IV) first increased with the increase of extraction time, and then reached a plateau after 25 minutes. Therefore, an extraction time of 30 minutes was selected for further study.

In addition, the effect of temperature on extraction was investigated by varying the temperature between $20^{\circ} \mathrm{C}$ and $60^{\circ} \mathrm{C}$. The results showed that the extraction efficiency increased from $20^{\circ} \mathrm{C}$ to $40{ }^{\circ} \mathrm{C}$, but decreased with an increase in temperature higher than $50{ }^{\circ} \mathrm{C}$. Based on these results, further experiments were carried out at $45^{\circ} \mathrm{C}$.

\section{Stirring Rate}

Effective mixing of the aqueous and organic phase is essential for mass-transfer of the analyte from aqueous to organic phase and reducing the extraction time. Thus, the effect of stirring rate on extrac-

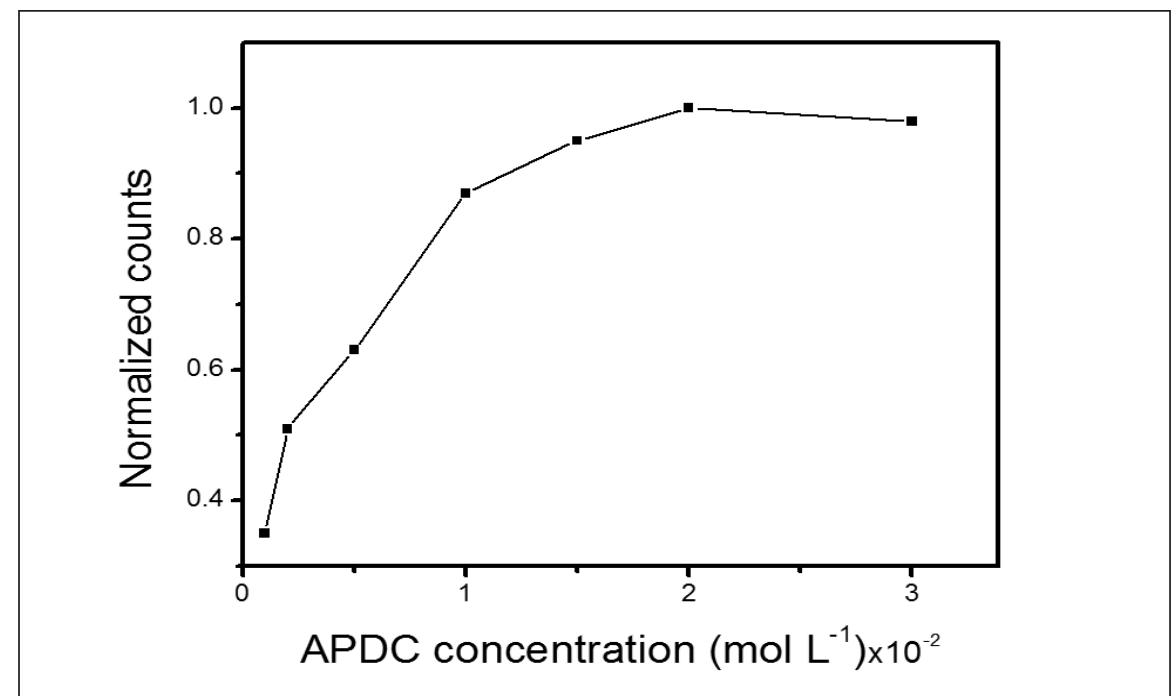

Fig. 3. Effect of APDC concentration on SFODME. Se(IV): $1.0 \mathrm{ng} m L^{-1}$.

tion was studied in the range of 200-1200 rpm. The experimental results showed that the signal intensity of the analyte increased rapidly by increasing the stirring rate from 200 to $800 \mathrm{rpm}$. However, the increasing rate of signal intensity decreased when the stirring rate exceeded $1000 \mathrm{rpm}$ owing to the loss of floating solvent drop. Therefore, a stirring rate of $900 \mathrm{rpm}$ was used for analysis.

\section{Effect of Sample Volume}

An important parameter which affects preconcentration and separation of the analytes is sample volume. Therefore, the effect of sample volume on the extraction of $0.1 \mathrm{ng}$ Se(IV) in different sample volumes ranging from $2.0-30 \mathrm{~mL}$ was investigated. The results indicated that the extraction was quantitative (recovery $>90 \%$ ) with the aqueous phase volume in the range of $2.0-15 \mathrm{~mL}$, and then decreased with a further increase in sample volume. Considering the two factors of analysis time and fold enrichment, a 10-mL sample volume was used for the analysis of the real samples. Based on the organic phase volume of $20 \mu \mathrm{L}$ and the maximum sample volume of $10 \mathrm{~mL}$, an enrichment factor of
500 was obtained for Se(IV) in this study.

\section{Effect of Diverse Ions}

In order to evaluate the application of this method for the determination of Se(IV) and Se(VI) in real water samples, the effect of various cations and anions on the extraction and determination of the analyte was investigated. The tolerance limit of coexisting ions is defined as the largest amount making variations of less than $10 \%$ error in the recovery of the analyte. The experimental results in Table II show that the selected ions were not found to interfere with the determination of $\mathrm{Se}(\mathrm{IV})$ and $\mathrm{Se}$ (VI) in the range of their concentration.

TABLE II Effect of Diverse Ions

\begin{tabular}{lr}
\hline Diverse Ions & $\begin{array}{c}\text { Concentration } \\
\text { Ratio }\end{array}$ \\
\hline $\mathrm{Na}^{+}, \mathrm{K}^{+}$ & 20,000 \\
$\mathrm{Ca}^{2+}, \mathrm{Mg}^{2+}$ & 10,000 \\
$\mathrm{Fe}^{3+}, \mathrm{Zn}^{2+}, \mathrm{Al}^{3+}, \mathrm{Cu}^{2+}$ & 500 \\
$\mathrm{SO}_{4}{ }^{2-}, \mathrm{SiO}_{3}{ }^{2-}, \mathrm{PO}_{4}{ }^{3-}$ & 10,000 \\
$\mathrm{Cl}^{-}, \mathrm{NO}_{3}{ }^{-}$ & 20,000 \\
\hline
\end{tabular}

${ }^{\mathrm{a}}$ Foreign ion / analyte. 


\section{Choice of Temperature Program}

To quantitatively vaporize the SeAPDC complex into the ICP, the temperature program of ETV was optimized and selected for this work. During the drying step, a drying temperature of $100^{\circ} \mathrm{C}$ and a drying time of 10 seconds were used for removal of the water vapor.

For removal of the extraction solvent from the furnace as much as possible without loss of analytes, the effect of charring temperature on signal intensity was studied in the range of $100-400{ }^{\circ} \mathrm{C}$. The results showed that the signal intensity of the analytes decreased obviously when the charring temperature was higher than $300{ }^{\circ} \mathrm{C}$ due to the thermal decomposition of the complex. In addition, the experimental results demonstrated that no significant influence was observed in the time range of 5-30 seconds. Based on the above facts, a charring temperature of $200{ }^{\circ} \mathrm{C}$ and a charring time of 30 seconds were used in this study.

With and without APDCP as a chemical modifier, the effect of the vaporization temperature on the signal intensity of Se(IV) was studied at the optimum time of 4 seconds. It is clear that the addition of APDC greatly affected the vaporization behavior of Se(IV), and its signal intensity reaches a plateau at a lower vaporization temperature of $1300{ }^{\circ} \mathrm{C}$. This illustrates that the chelating reaction between Se(IV) and APDC was complete. On the other hand, no plateau was found in the same temperature range in the absence of APDC. Moreover, the Se(IV) signal intensity is much more intense with APDC than without it, despite the application of a much higher vaporization temperature in the latter case. Thus, a vaporization temperature of $1300^{\circ} \mathrm{C}$ was chosen in this work.

\section{Calibration, Precision, and Detection Limit}

The linear dynamic range of the calibtation curve for this method covered three orders of magnitude with a correlation coefficient better than 0.9948 . The detection limits of Se(IV) and Se(VI), based on three times the standard deviation of the blank solution, were $0.26 \mathrm{pg} \mathrm{mL}^{-1}$ and $0.34 \mathrm{pg} \mathrm{mL}^{-1}$, respectively. The precision, expressed as the relative standard deviations obtained for nine replicate determinations at $0.1 \mathrm{ng} \mathrm{mL}^{-1}$ analytes, were $4.0 \%$ and $5.2 \%$, respectively.

\section{Sample Analysis}

This method was used for the determination of Se(IV) and Se(VI) in natural water samples, including lake water, river water, and pool water. In addition, recovery experiments of different amounts of the analytes were carried out, and the results are summarized in Table III. It can be seen that the recoveries in the range of $93.2-106 \%$ are reasonably well for trace analysis.

For validation of this method, a certified reference material, GSB07-1253-2000 water sample, obtained from Institute for Reference Materials of SEPA (Beijing, P.R. China), was analyzed and the analytical results were in good agreement with the certified value (see Table IV).

\section{CONCLUSION}

In this study, a solidified floating organic drop microextraction (SFODME) method coupled with electrothermal vaporization (ETV)

TABLE III

Analytical Results of Se(IV) and Se(VI) in Water Samples

\begin{tabular}{|c|c|c|c|c|c|c|c|}
\hline \multirow[t]{2}{*}{ Sample } & \multicolumn{2}{|c|}{ Added $\left(\mathrm{ng} \mathrm{mL} L^{-1}\right)$} & \multicolumn{3}{|c|}{ Found $^{\mathrm{a}}\left(\mathrm{ng} \mathrm{mL}^{-1}\right)$} & \multicolumn{2}{|c|}{ Recovery (\%) } \\
\hline & $\mathrm{Se}(\mathrm{IV})$ & $\mathrm{Se}(\mathrm{VI})$ & $\mathrm{Se}(\mathrm{IV})$ & $\mathrm{Se}(\mathrm{VI})^{\mathrm{b}}$ & Total & $\mathrm{Se}(\mathrm{IV})$ & $\mathrm{Se}(\mathrm{VI})$ \\
\hline \multirow[t]{3}{*}{ Pool Water } & 0 & 0 & $0.297 \pm 0.017$ & $0.135 \pm 0.009$ & $0.432 \pm 0.031$ & - & - \\
\hline & 0.5 & 0.5 & $0.763 \pm 0.049$ & $0.665 \pm 0.037$ & $1.428 \pm 0.084$ & 93.2 & 106 \\
\hline & 1.0 & 1.0 & $1.327 \pm 0.084$ & $1.082 \pm 0.061$ & $2.409 \pm 0.152$ & 103 & 94.7 \\
\hline \multirow[t]{3}{*}{ East Lake Water } & 0 & 0 & $0.173 \pm 0.012$ & $0.102 \pm 0.007$ & $0.275 \pm 0.015$ & - & - \\
\hline & 0.5 & 0.5 & $0.644 \pm 0.041$ & $0.607 \pm 0.053$ & $1.251 \pm 0.095$ & 94.2 & 101 \\
\hline & 1.0 & 1.0 & $1.108 \pm 0.091$ & $1.093 \pm 0.069$ & $2.201 \pm 0.172$ & 93.5 & 99.1 \\
\hline \multirow[t]{3}{*}{ Yangtse River Water } & 0 & 0 & $0.348 \pm 0.015$ & $0.137 \pm 0.008$ & $0.485 \pm 0.039$ & - & - \\
\hline & 0.5 & 0.5 & $0.822 \pm 0.054$ & $0.627 \pm 0.041$ & $1.449 \pm 0.062$ & 94.8 & 98.0 \\
\hline & 1.0 & 1.0 & $1.304 \pm 0.067$ & $1.116 \pm 0.084$ & $2.420 \pm 0.115$ & 95.6 & 97.9 \\
\hline
\end{tabular}

${ }^{a}$ Mean value \pm standard deviation, $n=5$.

${ }^{\mathrm{b}}$ Calculated value. 


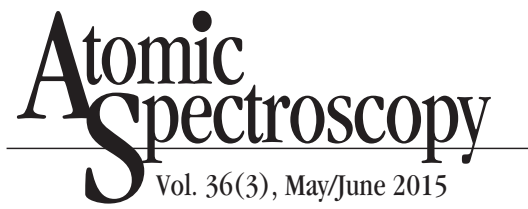

TABLE IV

Analytical Results of Se(IV) and Se(VI)

in Environmental Reference Sample

\begin{tabular}{lccccc}
\hline Sample & Element & $\begin{array}{c}\text { Added } \\
\left(\mathrm{ng} \mathrm{mL}^{-1}\right)\end{array}$ & $\begin{array}{c}\text { Found }^{\mathrm{a}} \\
\left(\mathrm{ng} \mathrm{mL}^{-1}\right)\end{array}$ & $\begin{array}{c}\text { Certified } \\
\left(\mathrm{ng} \mathrm{mL}^{-1}\right)\end{array}$ & $\begin{array}{c}\text { Recovery } \\
(\%)\end{array}$ \\
\hline $\begin{array}{l}\text { GSB07-1253-2000 } \\
\text { Water }\end{array}$ & $\mathrm{Se}(\mathrm{IV})$ & 0 & - & - & - \\
& & 5.0 & $4.74 \pm 0.37$ & - & 94.8 \\
& $\mathrm{Se}(\mathrm{VI})$ & 0 & $5.29 \pm 0.51$ & $5.0^{\mathrm{b}}$ & - \\
& & 5.0 & $10.2 \pm 0.73$ & - & 104 \\
\hline
\end{tabular}

${ }^{a}$ Mean value \pm standard deviation, $n=3$.

${ }^{\mathrm{b}}$ Reference value.

for the preconcentration, separation, and determination of Se(IV) and $\mathrm{Se}(\mathrm{VI})$ in natural water samples by inductively coupled plasma mass spectrometry (ICP-MS) is described. APDC and 1-undecanol were selected as a chelating reagent and an extraction solvent in SFODME, respectively. At the same time, APDC acted as a chemical modifier for subsequent experiments in the ETV-ICP-MS step. The experimental results showed that the Se(IV)APDC complex can be extracted effectively with 1-dodecanol and vaporized quantitatively by ETV into the ICP-MS. In addition, this method provides advantages such as high enrichment factor, low detection limit, simple operation, good precision and accuracy. With these advantages, the method has great potential to be used for the separation, preconcentration, and determination of trace/ultra-trace elements and their speciation in real samples.

\section{ACKNOWLEDGMENT}

Financial support from the Nature Science Foundation and the Education Department Foundation of Hubei Province in China is gratefully acknowledged.

Received November 17, 2014.

\section{REFERENCES}

1. T. Ferri, C.D. Luca, and L. Ticconi, Anal. Lett. 34, 975 (2001).

2. N. Zhang, N. Fu, Z. Fang, Y. Feng, and L. Ke, Food Chem. 124, 1185 (2011).

3. M.P. Rayman, Proc. Nutr. Soc. 61, 213 (2002).

4. K. Chandrasekaran, M. Ranjit, D. Karunasagar, and J. Arunachalam, At. Spectrosc. 29, 129 (2008).

5. H.E Gangher, O.A. Levander, and C.A. Baumann, J. Nutr. 88, 55 (1996).

6. S.V. Rao and J. Arunachalam, At. Spectrosc. 27, 165 (2006).

7. M. Soylak and N. Kizil, At. Spectrosc. 34, 216 (2013).

8. Z. Wang, M. Song, Q. Ma, H. Ma, and S. Liang, Microchim. Acta 34, 95 (2000).

9. E. Kilinc, F. Aydin, and M.Z. Duz, At. Spectrosc. 33, 173 (2012).

10. S. Chen, X. Guo, Y. He, and D. Lu, At. Spectrosc. 34, 113 (2013).

11. H. Matusiewicz and M. Slachcinski, Microchem. J. 102, 61 (2012).

12. Y. Zhao, J. Zheng, M. Yang, G. Yang, Y. Wu, and F. Fu, Talanta 84, 983 (2011).

13. Z. Joanna, M. Henryk, P. Halina, and C. Ewelina, Food Chem. 142, 178 (2014).

14. G. Mahnaz, Y. Yadollah, and E. Ali, Microchim. Acta 180, 519 (2013).

15. P. Liang, J. Yu, E. Yang, and L. Peng, At. Spectrosc. 35, 85 (2014).
16. T. Somayeh and A.T. Mohammad, Microchim. Acta 173, 249 (2011).

17. R.E. Rivas, I. López-García, and M. Hernández-Córdoba, Anal. Meth. 2, 225 (2010).

18. J. Ma, J. Zhang, X. Du, X. Lei, and J. Li, Microchim. Acta 168, 153 (2010).

19. I. Durukan, C.A. Şahin, and S. Bektaş, Microchem. J. 98, 215 (2011).

20. S. Chen, S. Zhu, and D. Lu, At. Spectrosc. 35, 1 (2014).

21. X. Guo, M. He, B. Chen, and B. Hu, Talanta 94, 70 (2012).

22. S. Chen, H. Zhang, and B. Ruan, At. Spectrosc. 30, 143 (2008).

23. S. Huang and S. Jiang, Anal. Meth. 2, 1310 (2010).

24. Y. Yi, S. Jiang, and A.C Sahayam, J. Anal. At. Spectrom. 27(3), 426 (2012).

25. Y. Yi, S. Wu, and S. Jiang, At. Spectrosc. 34, 39 (2013).

26. S. Mei, L. Guijian, and W. Qianghua, Food Chem. 141(1), 66 (2013).

27. M.R.K. Zanjani, Y. Yamini, S. Shariati, and J.A. Jonsson, Anal. Chim. Acta 585(2), 286 (2007). 